\title{
Fármacos propuestos para el manejo de pacientes adultos con enfermedad por coronavirus (COVID-19)
}

\author{
Karen Gómez ${ }^{1}$ \\ ${ }^{1}$ Affiliation not available
}

August 17, 2020

\section{Resumen:}

La pandemia por coronavirus COVID-19 ha representado una enorme crisis de salud global. La industria farmacéutica actualmente está desarrollando nuevos tratamientos dirigidos al COVID-19 y en ausencia de algún fármaco aprobado por la FDA dirigido al tratamiento específico de COVID-19 la comunidad médica está probando una variedad de terapias que poseen efectos antivirales e inmunomoduladores para prevenir y tratar esta enfermedad. Algunas de las drogas implementadas en el tratamiento de pacientes con enfermedad por Coronavirus han sido recientemente desarrolladas y aún se encuentran en proceso investigación, y algunas otras son drogas ampliamente utilizadas en infecciones por SARS-COV y MERS-COV cuyos mecanismos de acción pueden resultar beneficiosos en el tratamiento de COVID-19, estos datos han sido descritos en estudios observacionales y están siendo utilizados anecdóticamente en base a evidencia in vitro o extrapolada. Es importante reconocer que no hay datos controlados que respalden el uso de ninguno de estos agentes y su eficacia para el COVID-19 es desconocida por lo que se están llevando a cabo una serie de estudios de investigación para el desarrollo de fármacos dirigidos al tratamiento antiviral del COVID-19. Por esta razón la práctica médica exige la búsqueda sistemática de información actualizada para dar respuestas a las interrogantes que surgen en el ejercicio de la actividad profesional durante el tratamiento de pacientes con infección por CODIV-19, para lo cual la medicina basada en la evidencia tiene una amplia aplicación en la toma de decisiones terapéuticas implementadas en el tratamiento de la enfermedad por COVID-19.

Palabras clave: Coronavirus, Infecciones por coronavirus, Enfermedad por Coronavirus 2019-nCoV, Terapeutica

\section{INTRODUCCIÓN:}

La enfermedad por COVID-19 (Coronavirus Disease-2019) es una emergencia de salud pública de interés internacional donde aproximadamente el $15 \%$ de las personas contagiadas evolucionaran a una forma severa de la enfermedad requiriendo cuidados hospitalarios. Actualmente no se cuenta con un tratamiento farmacológico con comprobada especificidad para el tratamiento de COVID-19 por lo que grupos multidisciplinarios de diferentes organizaciones han creado protocolos de tratamiento basados en la actual evidencia de drogas que han resultado beneficiosas en el tratamiento de COVID-19 por lo que el presente ensayo tiene como objetivo conocer cuáles son los fármacos actualmente propuestos que son utilizados en el manejo de pacientes adultos con enfermedad por coronavirus, para ello se realizó la revisión actual de la literatura científica disponible acerca de las opciones terapéuticas en el manejo de pacientes por enfermedad de coronavirus (COVID-19) en la base de datos PUBMED publicados en el año 2020 utilizando los siguientes 
términos de búsqueda:COVID-19 OR CORONAVIRUS AND "DRUG THERAPY" AND COVID-19 OR CORONAVIRUS AND "COMBINATION DRUG THERAPY", tras la cual se identificaron 66 artículos de los cuales se seleccionaron 16 estudios por ser relevantes para el objetivo de esta revisión, los cuales contenían recomendaciones de diversas sociedades profesionales. Así mismo se realizó la revisión de los actuales lineamientos de tratamiento para pacientes hospitalizados con enfermedad por COVID-19 proporcionados por el ministerio de salud de El Salvador (MINSAL) .La importancia del conocimiento de este tema radica en que la práctica médica exige la búsqueda sistemática de información actualizada a través de la investigación para dar respuestas a las interrogantes que surgen en el ejercicio de la actividad profesional durante el tratamiento de pacientes con infección por CODIV-19 que necesitan manejo hospitalario, para lo cual la medicina basada en la evidencia tiene una amplia aplicación en la toma de decisiones terapéuticas, en especial dentro del manejo, prevención y control de las posibles complicaciones asociadas a infección por COVID-19.

\section{DESARROLLO TEMÁTICO:}

Desde la aparición de casos de infección por coronavirus (COVID-19) existe un gran interés en el desarrollo de una terapia farmacológica efectiva ya que en la actualidad no existe un protocolo de tratamiento registrado que sea específico para el manejo de la infección por COVID-19. Sin embargo, existen reportes anecdóticos y datos de estudios acerca del uso de diversos fármacos los cuales han resultado efectivos en el tratamiento de la infección por coronavirus.

Dentro de los grupos de fármacos propuestos para el manejo de pacientes con enfermedad por COVID-19 se encuentran los siguientes:

- Antivirales

- Aminoquinolinas

- Glucocorticoides

- Antibióticos

- Agentes inmunomoduladores

- Hemocomponentes

- Vitaminas y Oligoelementos

\section{ANTIVIRALES}

Los antivirales más ampliamente investigados para el tratamiento de COVID-19 en ensayos clínicos o en estudios invitro son el análogo de los nucleótidos Remdesivir y los inhibidores de la proteasa Lopinavir/Ritonavir.

\section{Remdesivir}

Remdesivir es un nuevo análogo de los nucleótidos que posee actividad en el síndrome respiratorio agudo severo por coronavirus 2 ( SARS-Cov-2) in vitro (1). En Estados Unidos, la Food and Drug Administration (FDA) ha emitido la autorización de su uso para pacientes hospitalizados tanto como niños como adultos con enfermedad severa por COVID-19 definida como una saturación de Oxigeno SpO2 [?] 94\% al aire ambiente, requerimiento de oxigeno suplementario, ventilacion mecanica u oxigenacion por membrana extracorporea (2)

Remdesivir actua como un analogo del trifosfato de adenosina y compite por su incorporacion dentro de las cadenas de ARN del coronavirus 2 ( SARS-CoV) ARN polimerasa dependiente, dando como resultado el retraso de la conformacion de la cadena lateral del ARN durante su replicacion.(2) 
Diversos estudios comparan su eficacia frente a placebos en el tratamiento de pacientes con COVID-19 y evidencia de afeccion pulmonar en donde segun reportes preliminares el uso de Remdesivir resulto en un tiempo de recuperacion mas rapido, permitiendo el alta de los pacientes o la continuacion de su hospitalizacion sin necesidad de oxigeno suplementario aunque no se constato disminucion del indice de mortalidad significativa ni una diferencia en el tiempo de eliminacion viral (3)

La dosis recomendada y la via de administracion durante el tratamiento con Remdesivir es de $200 \mathrm{mg}$ via IV en el primer dia de tratamiento seguido de $100 \mathrm{mg}$ al dia por 10 dias en total en pacientes con ventilacion mecanica u oxigenacion por membrana extracorporea y 5 dias en total para otros pacientes.(3)

Por ser un farmaco experimental el perfil de efectos adversos de Remdesivir no se conoce a profundidad. Se puede afirmar que la elevacion de enzimas hepaticas ALT y AST es el evento adverso mas caracteristico. Por lo que se recomienda estimar sus niveles antes de iniciar el tratamiento, asi como el estado de la funcion renal, suspendiendo o no iniciando la administracion de Remdesivir si se observa un nivel de AST mas de 5 veces el limite superior normal o una tasa de filtrado glomerular menor de $30 \mathrm{ml} / \mathrm{min}$. (4)

\section{Lopinavir/ Ritonavir}

El farmaco Lopinavir/ Ritonavir es una combinacion de dos farmacos antirretrovirales inhibidores de la proteasa, empleados en el tratamiento de la infeccion por VIH en adultos y neonatos mayores de 14 dias de nacimiento donde Ritonavir funge como potenciador farmacocinetico de Lopinavir.(4)

Actualmente el posible uso de Lopinavir/Ritonavir en el contexto de infeccion por COVID-19 se basa en datos anecdoticos contenidos en estudios clinicos donde se ha demostrado que el farmaco Lopinavir/Ritonavir posee actividad in vitro ante los virus SARS-CoV y MERS-Cov (5) A pesar de ello el uso de Lopinavir/Ritonavir en el tratamiento de COVID-19 se encuentra bajo investigacion y su eficacia asi como su seguridad no ha sido establecida.

Algunos estudios demuestran que pacientes infectados por el virus SARS-CoV tratados con este farmaco presentaban un riesgo menor de sindrome respiratorio agudo apoyando de esta manera su incorporacion dentro de guias de tratamiento para infeccion por COVID-19 en paises como China en las cuales se sugiere una dosis de $200 \mathrm{mg} / 50 / \mathrm{tab}, 2$ tabletas via oral cada 12 horas por no mas de 10 dias (6) .

En el Salvador el farmaco Lopinavir/Ritonavir se emplea en el tratamiento de Neumonia grave y neumonia grave asociada a ventilacion mecanica en dosis de 400/100 mg (2 comprimidos de 200/50 mg) VO 2 veces al dia durante 14 dias $(7)$

Dentro de los efectos adversos que pueden presentarse durante el tratamiento con Lopinavir/Ritonavir se encuentran nauseas, vomitos, diarrea, prolongacion del intervalo QT, hepatotoxicidad y por ser un farmaco con una accion inhibitoria de CYP3A potente, muchos medicamentos metabolizados por esta enzima pueden causar toxicidad severa.(8)

\section{AMINOQUINOLINAS}

Los farmacos Hidroxicloroquina y Cloroquina son medicamentos pertenecientes al grupo de farmacos aminoquinolinas que generalmente son ampliamente utilizados para el tratamiento de enfermedades cronicas como Lupus eritematoso, Artritis reumatoide, patologias dermatologicas e infecciones como la malaria y el cual ha mostrado actividad antiviral contra el virus del sindrome respiratorio agudo severo (SARS-COV-2) y una respuesta antiinflamatoria en un pequeno grupo de estudios clinicos controlados. 
Se ha demostrado que la hidroxicloroquina (un analogo de la cloroquina) tiene una actividad anti-SARS-CoV in vitro. El perfil de seguridad clinica de hidroxicloroquina es mejor que el de cloroquina (durante el uso a largo plazo) y permite una dosis diaria mas alta.

Debido a las similitudes del COVID-19 con el SARS-COV muchos investigadores han propuesto el uso de hidroxicloroquina y cloroquina en la actual pandemia por COVID-19 ya que en China se realizaron diversos ensayos clinicos donde probaron la eficacia y seguridad de estos medicamentos en el tratamiento de neumonia asociada a COVID-19 en mas de 10 hospitales de diferentes ciudades y evidenciaron que el fosfato de cloroquina inhibia la exacerbacion de la neumonia mejorando los hallazgos radiologicos pulmonares, promovia una seroconversion viral negativa y un acortamiento del curso de la enfermedad, por lo que actualmente es una de las drogas recomendadas para su inclusion dentro de la nueva version de las guias de prevencion, diagnostico y tratamiento de neumonia causada por COVID-19 emitida por la comision nacional de salud de la Republica Popular de China, por su parte la FDA ha emitido la autorizacion del uso de fosfato de cloroquina proporcionado por la Reserva Nacional Estrategica para tratar a adultos y adolescentes que pesan $50 \mathrm{~kg}$ o mas y que estan hospitalizados con COVID-19, para quienes no hay un estudio clinico disponible e o su participacion no es posible. (9)

La dosis optima y la duracion del tratamiento para COVID-19 con fosfato de cloroquina se desconoce pero la FDA sugiere el uso 1 gramo de fosfato de cloroquina en el primer dia de tratamiento y luego 500 miligramos al dia durante cuatro a siete dias de tratamiento en total, basado en la evolucion clinica del paciente (9)

El uso de los farmacos hidroxicloroquina y cloroquina debe ser estrictamente monitorizado por la posibilidad de efectos adversos ya que estos pueden generar a nivel cardiaco efectos como prolongacion del complejo QT evidenciado por electrocardiograma, arritmias ventriculares, cardiomiopatias, torasades de pointes; asi mismo puede generar reacciones adversas a nivel oftalmologico y otologico asociadas a las dosis acumulativa del farmaco presentandose como alteraciones corneales, disminucion de la agudeza visual, sordera y tinnitus respectivamente.

Es importante recalcar que a pesar de las recomendaciones sobre el uso de hidroxicloroquina los datos para respaldar el uso de estos farmacos para tratamiento de patologia pulmonar asociada a COVID-19 son limitados e inconclusos y no se ha demostrado la total eficacia y seguridad el farmaco para este proposito por lo que se encuentra en fase de estudio para la generacion de recomendaciones de su uso con evidencia cientifica solida.

\section{GLUCOCORTICOIDES}

El uso de glucocorticoides orales y parenterales fue muy amplio durante la epidemia por el virus SARS-CoV en el ano 2003 en Hong Kong China debido a datos anecdoticos que respaldaban su uso y a hallazgos radiologicos e histopatologicos pulmonares de pacientes con sindrome respiratorio agudo por el virus SARS-COV.

Un estudio de cohorte durante este brote epidemico demostro que la administracion de altas dosis en pulsos de metilprednisolona se asocio a una mejoria clinica de pacientes con SARS y a la reduccion en los niveles de ciertas citocinas proinflamatorias posterior a cinco u ocho dias de tratamiento(10). Se emplearon diferentes regimenes de tratamiento en donde los principales incluian la administracion de metilprednisolona 1-2 $\mathrm{mg} / \mathrm{kg} /$ dia o $2-4 \mathrm{mg} /$ dia cada 8 horas via IV, seguido de prednisolona oral con dosis dispares durante un tiempo que variaba de acuerdo a la evolucion clinica del paciente o la administracion de metilprednisolona $500 \mathrm{mg}$ al dia via IV durante 5 dias seguido de la administracion de prednisolona $50 \mathrm{mg}$ cada 12 horas, con reduccion de 20-30 mg diarios a partir del dia 21 de tratamiento segun evaluacion clinica (11)

Debido a su amplia utilizacion durante la epidemia por SARS-COV en pacientes con sintomas respiratorios severos se ha sugerido el uso de este farmaco para el tratamiento de pacientes con enfermedad severa por 
COVID-19. Actualmente el centro para control y prevencion de enfermedades (CDC) y la organizacion mundial de la salud (OMS) proponen que los glucocorticoides no deben ser administrados rutinariamente a pacientes con COVID-19 al no ser que exista una indicacion como asma o exacerbacion de enfermedad pulmonar obstructiva cronica, shock septico refractario e insuficiencia adrenal. Basados en datos que reportan un potencial beneficio de los glucocorticoides en pacientes con moderada a severa dificultad respiratoria aguda la sociedad de medicina critica (SCCM) provee una recomendacion provisional, debil en favor del uso de estos farmacos en pacientes con COVID-19 y dificultad respiratoria aguda, y en caso de ser utilizados se recomienda administrarlos dentro de los primeros 14 dias del cuadro y las dosis utilizadas deben ser bajas con curso de tratamiento corto por ejemplo dexametasona $20 \mathrm{mg} / \mathrm{dia}$ IV por 5 dias luego $10 \mathrm{mg}$ a vez al dia por 5 dias. A pesar de ello Los efectos clinicos y terapeuticos de la terapia de glucocorticoides sistemicos en pacientes con COVID-19 no se encuentran del todo claro por lo que se requieren mas investigaciones acerca de sus beneficio en el contexto de infeccion por COVID-19.

En el Salvador el farmaco metilprednisolona es el glucocorticoide utilizado en pacientes con neumonia grave con ventilacion mecanica a dosis de $250 \mathrm{mg}$ IV en bolo y despues a los dias $2-7 \mathrm{mg} / \mathrm{kg} / \mathrm{dia}$ IV (9)

Las posibles complicaciones del uso de glucocorticoides como inmunosupresion profunda con la posible emergencia de infeccion invasiva de origen viral, bacteriana o micotica, osteonecrosis y psicosis pueden ocurrir con una terapia prolongada con altas dosis de glucocorticoides por lo que se sugiere la vigilancia de la aparicion de las mismas.

\section{ANTIBIOTICOS}

El farmaco azitromicina es un antibiotico de amplio espectro del grupo de las macrolidos que actua contra varias bacterias Gram positivas y Gram negativas mycoplasma pneumoniae, Treponema pallidum, Chlamydia y Mycobacterium avium complex.

Azitromicina actua en las bacterias por medio de su union en la sub unidad ribosomal $50 \mathrm{~S}$ e inhibe la sintesis de ARN mensajero, ademas ha mostrado actividad invitro contra el virus zika y ebola, el posible mecanismo de accion contra los virus no esta del todo claro por lo que se planteo la hipotesis de que los macrolidos podrian inhibir al virus sincitial respiratorio por medio de la reduccion de la expresion del receptor de proteina de fusion, isoforma A activada de la familia Rashomologus (Rho) y la inhibicion de la posterior activacion de Rho quinasa en celulas epiteliales de las vias respiratorias.(12)

De acuerdo a datos anecdoticos de experiencias en China y en paises europeos en el tratamiento de infeccion por COVID-19 se ha sugerido que el uso combinado de azitromicina e hidroxicloroquina podria resultar beneficioso en la evolucion clinica de los pacientes con dicha infeccion. Un estudio de cohorte realizado en China demostro que el uso de azitromicina en combinacion con hidroxicloquina se asocio a una mejoria clinica superior en comparacion con la evolucion clinica de otros pacientes hospitalizados y a una disminucion relativamente rapida de la carga viral de ARN evaluada por PCR, que fue aun mas rapida cuando se evaluo por cultivo.(13)

Tanto la azitromicina como la hidroxicloroquina estan asociadas con la prolongacion del complejo QT, y el uso combinado puede potenciar este efecto adverso. En un gran estudio observacional de pacientes hospitalizados con COVID-19 en Nueva York, la tasa de mortalidad ajustada entre los que recibieron azitromicina mas hidroxicloroquina fue similar en comparacion con aquellos que no recibieron ninguno de los agentes, pero la tasa de paro cardiaco fue mayor(14)

La evidencia demuestra un efecto beneficioso de la administracion conjunta de hidroxicloroquina con azitromicina en el tratamiento de COVID-19 y su potencial efectividad en la reduccion temprana de contagio. Dada la urgente necesidad terapeutica de controlar enfermedad por COVID-19 con medicamentos efectivos 
y seguros, existe una necesidad urgente de evaluar mas esta estrategia para tratar a los pacientes en una etapa temprana de la enfermedad antes que surjan complicaciones respiratorias graves e irreversibles, y para disminuir la duracion de la enfermedad y su propagacion.

\section{AGENTES INMUNOMODULADORES}

En la infeccion severa por COVID-19 se presenta una marcada elevacion de marcadores infamatorios y una produccion elevada de citocinas inflamatorias lo que se ha asociado a una evolucion critica de la enfermedad. Por ello se ha propuesto que el bloqueo de las vias inflamatorias puede prevenir la progresion de la enfermedad.

Actualmente la tormenta de citocinas mediada por la sobre produccion de citocinas proinflamatorias se ha observado en un gran numero de pacientes gravemente enfermos por COVID-19. Los pacientes que presentan este fenomeno progresan a colapso cardiovascular, disfuncion multiorganica y muerte.

Los corticoesteroides como el farmaco Metilprednisolona es uno de los agentes ampliamente utilizados para frenar la produccion excesiva de citocinas, sin embargo el tratamiento con altas dosis de corticoesteroides por un periodo de tiempo prolongado se asocia a la aparicion de efectos adversos graves. En un intento de proporcionar un efecto ahorrador de corticoesteroides, el uso del farmaco Tocilizumab fue recomendado en pacientes con infeccion COVID-19 en estado critico para prevenir la tormenta de citocinas basado en el conocimiento del rol de la interleucina 6 en esta enfermedad.

La IL-6 es una citocina que juega un rol importante en la respuesta inflamatoria e inmunologica. La experiencia clinica en China en el manejo de COVID-19 sugiere que la IL-6 es una de las citocinas mas importantes involucradas en la tormenta de citocinas desencadenada por este virus. Por esta razon el farmaco Tocilizumab es recomendado en pacientes gravemente enfermos.

Tocilizumab es un antagonista del receptor de interleucina 6. Endogenamente la IL-6 induce una respuesta inflamatoria y es mediadora de una gran variedad de respuestas inmunologicas, la inhibicion de los receptores de la IL-6 por este farmaco conduce a una reduccion en la produccion de citoquinas y reactantes de fase aguda.

En estudios multicentricos y observacionales se ha descrito que el uso de Tocilizumab dentro de los primeros 6 dias de admision hospitalaria de pacientes con infeccion severa por COVID-19 que presentaban resultados de laboratorio que sugerian un estado pro-inflamatorio y pro trombotico la administracion de este farmaco se asocio a una disminucion de la proteina $\mathrm{C}$ reactiva, dimero $\mathrm{D}$, niveles de ferritina y una mejora en la relacion entre la presion parcial de oxigeno $(\mathrm{Pa} 02)$ y la fraccion de oxigeno inspirado $(15,16)$ por lo que su uso en pacientes adultos hospitalizados con infeccion severa por COVID-19 Tocilizumab parece ser una opcion segura basado en los hallazgos clinicos secundarios al uso de esta terapia y debe ser mas ampliamente estudiada para confirmar el beneficio definitivo del uso de antagonistas de receptores de la IL-6.

Actualmente en El Salvador el farmaco Tocilizumab esta indicado en casos de neumonia grave a dosis de 600 mg IV, dosis unica para pacientes mayores de $75 \mathrm{~kg}$ y $400 \mathrm{mg}$ IV dosis unica para pacientes menores de 75

kilogramos. En pacientes con neumonia grave con ventilacion mecanica se asocia el uso de metilprednisolona a las dosis anteriores de Tocilizumab. (7)

\section{HEMOCOMPONENTES}

El plasma convaleciente se refiere al plasma sanguineo de una persona que se ha recuperado de una infeccion. Es un medio de transferencia de anticuerpos para proporcionar inmunidad pasiva (a traves de anticuerpos 
neutralizantes o posiblemente otros mediadores inmunes dirigidos contra el patogeno infeccioso) hasta que el individuo pueda desarrollar una respuesta inmune activa, con la esperanza de que los resultados clinicos puedan mejorar en el receptor (15)

El plasma convaleciente no esta disponible de manera rutinaria, ni es un producto con licencia de la FDA; en cambio, se ha puesto a disposicion de agentes especificos en momentos de epidemias o pandemias de enfermedades. Una vez que ha disminuido una epidemia, es probable que el plasma convaleciente no este disponible.

Dentro del uso mas reconocido del plasma convaleciente destaca el llevado a cabo durante la epidemia por SARS-CoV en el ano 2003 donde secundario a su uso hubo una reduccion de la mortalidad (del orden de 7 a 23 por ciento menos de mortalidad). Asi mismo se asocio a una estancia hospitalaria reducida, especialmente cuando se administro plasma convaleciente dentro de las dos semanas posteriores al inicio de los sintomas.

Ademas se ha observado que el plasma convaleciente puede acelerar la erradicacion del virus, aumentar el numero de linfocitos plasmaticos y celulas Natural Killer (NK), reducir el nivel de acido lactico plasmatico y mejorar el funcionamiento renal (16)

Durante la pandemia de la enfermedad por coronavirus 2019-2020 (COVID-19), los centros comunitarios de sangre en los Estados Unidos y en todo el mundo estan estableciendo programas para que las personas recuperadas donen plasma convaleciente. El plasma se puede obtener mediante aferesis o donacion de sangre completa; se prefiere la aferesis porque esto optimiza el rendimiento (se pueden obtener de dos a cuatro unidades de plasma por donacion de aferesis) (15)

El plasma convaleciente de individuos que se han recuperado de COVID-19 puede proporcionar un beneficio clinico a los pacientes con COVID-19 activo cuando se administra en etapas tempranas de la enfermedad. En un ensayo abierto de China de 103 pacientes con COVID-19 grave o potencialmente mortal, la adicion de plasma convaleciente al tratamiento estandar mejoro la tasa de eliminacion del ARN viral en comparacion con el tratamiento estandar solo, pero no hubo diferencias estadisticamente significativas en el Tasas generales de mejoria clinica (52 versus 43 por ciento) o supervivencia (84 versus 76 por ciento) (17)

Estudios adicionales de plasma convaleciente estan en curso y en los Estados Unidos, la FDA tambien esta facilitando la evaluacion de la globulina hiperinmune para pacientes con COVID-19 asi como la investigacion del plasma convaleciente como opcion terapeutica por su parte La Cruz Roja Americana apoya los esfuerzos de la FDA y se compromete a ayudar con las recolecciones de plasma de pacientes recuperados con 9 COVID-19 cuidadosamente seleccionados para permitir un acceso rapido al tratamiento para los pacientes mas gravemente enfermos.

En El Salvador el uso del plasma convaleciente esta aprobado para el tratamiento de pacientes con infeccion por COVID-19 activo en estado critico o grave asociado o no a complicaciones y en pacientes que no estan en estado grave o critico, pero si en estado de inmunodepresion; y cuya progresion es muy rapida y afecta a los pulmones (16) La dosis del tratamiento con plasma convaleciente es [?] $400 \mathrm{~mL}$ para una infusion, o [?] $200 \mathrm{~mL}$ cada 12 horas por infusion para varias infusiones durante 2 a 3 dias, dichas dosis deben ser administradas en un periodo de 60 minutos.

Entre los efectos adversos mas frecuentes asociadas a la administracion de plasma convaleciente estan : Reacciones de transfusion hemolitica, reacciones febriles no hemoliticas, reacciones alergicas que van desde la urticaria hasta la anafilaxia, reacciones septicas, lesion pulmonar aguda relacionada con la transfusion, sobrecarga circulatoria, transfusion asociada a enfermedad de injerto contra huesped, purpura posterior a la transfusion (16) 


\section{VITAMINAS Y OLIGOELEMENTOS:}

\section{Vitamina D}

A traves de varios mecanismos, la vitamina D puede reducir el riesgo de infecciones. Esos mecanismos incluyen inducir catelicidinas y defensinas que pueden reducir las tasas de replicacion viral y reducir las concentraciones de citocinas proinflamatorias que producen la inflamacion que dana el revestimiento de los pulmones, lo que lleva a la neumonia, asi como el aumento de las concentraciones de citocinas antiinflamatorias. Varios estudios observacionales y ensayos clinicos informaron que la suplementacion con vitamina $\mathrm{D}$ redujo el riesgo de influenza. La evidencia que respalda el papel de la vitamina $\mathrm{D}$ en la reduccion del riesgo de COVID-19 incluye que el brote ocurrio en invierno, un momento en que las concentraciones de 25-hidroxivitamina D (25 (OH) D) son mas bajas; que el numero de casos en el hemisferio sur cerca del final del verano es bajo; se ha descubierto que la deficiencia de vitamina D contribuye al sindrome de dificultad respiratoria aguda; y que las tasas de letalidad aumentan con la edad y con la comorbilidad de enfermedades cronicas, las cuales estan asociadas con una menor concentracion de $25(\mathrm{OH})$ D. Para reducir el riesgo de infeccion, se recomienda que las personas con riesgo de influenza y / o COVID-19 consideren tomar 10,000 UI / d de vitamina D3 durante algunas semanas para aumentar rapidamente las concentraciones de $25(\mathrm{OH})$ $\mathrm{D}$, seguidas de $5000 \mathrm{UI} / \mathrm{re}$. El objetivo debe ser elevar las concentraciones de $25(\mathrm{OH}) \mathrm{D}$ por encima de 40-60 ng / ml (100-150 nmol / L). Para el tratamiento de personas que se infectan con COVID-19, pueden ser utiles dosis mas altas de vitamina D3. ) En El Salvador se recomienda el uso de vitamina D a dosis de 10000 UI diarias para pacientes mayores de 60 anos como parte del esquema de tratamiento para pacientes con neumonia moderada, grave y asociada a ventilacion mecanica. (9)

Se deben realizar ensayos controlados aleatorios y estudios de gran poblacion para evaluar estas recomendaciones.(18)

\section{Zinc}

En la decada de los anos 1960 se descubrio los efectos de la deficiencia de Zinc y como este es esencial para la funcion del sistema inmune. Los iones de zinc estan involucrados en la regulacion intracelular vias de senalizacion en celulas inmunes innatas y adaptativas. Las propiedades antiinflamatorias y antioxidantes del zinc han sido durante mucho tiempo documentadas, sin embargo, los mecanismos subyacentes aun no estan del todo claros. Una homeostasis equilibrada de zinc es crucial para defenderse contra la invasion de agentes patogenos o proteger el cuerpo humano contra un sistema inmunitario que reacciona de forma exagerada enfermedades autoinmunes, inflamacion cronica o alergias. En este sentido, el zinc puede considerarse como un guardian del sistema inmune, ya que la funcion adecuada de practicamente todas las celulas inmunes es altamente dependiente de zinc. El zinc inhibe la replicacion del virus y se ha probado en ensayos para el tratamiento del resfriado comun, mostrando una reduccion significativa en la duracion del resfriado a una dosis de [?] $75 \mathrm{mg} /$ dia (19) En El Salvador se recomienda el uso de ZINC a dosis $200 \mathrm{mg}$ diarios tabletas o jarabe para todas edades como parte del esquema de tratamiento para pacientes con neumonia moderada, grave y asociada a ventilacion mecanica. (9)

Algunos de los signos del consumo excesivo de zinc son: nauseas, vomitos, perdida del apetito, dolor abdominal, diarreas y dolores de cabeza. Puede obtener las cantidades recomendadas de zinc mediante el consumo de una variedad de alimentos, entre ellos: ostras, que son la mejor fuente de zinc; carnes rojas, carnes de ave, mariscos como cangrejo y langosta, y cereales para el desayuno fortificados, que son fuentes buenas de zinc; y frijoles, frutos secos, cereales integrales y productos lacteos, que tambien aportan el zinc.

\section{Vitamina $\mathbf{C}$}

La vitamina $\mathrm{C}$, tambien conocida como acido ascorbico, tiene propiedades antioxidantes y actualmente se estan desarrollando estudios que plantean la hipotesis que su administracion puede mejorar el pronostico de los pacientes con infeccion por COVID-19 en estado grave. 
En casos de sepsis, se produce el aumento de citoquinas causado por la misma, y hay acumulacion de neutrofilos en el tejido pulmonar, destruyendo los capilares alveolares. Los primeros estudios clinicos han demostrado que la vitamina $\mathrm{C}$ puede prevenir eficazmente este proceso. Ademas, la vitamina $\mathrm{C}$ puede ayudar a eliminar el liquido alveolar previniendo la activacion y acumulacion de neutrofilos y reduciendo el dano al canal epitelial alveolar. Al mismo tiempo, la vitamina $\mathrm{C}$ puede prevenir la formacion de trampas extracelulares de neutrofilos, que es un evento biologico de lesion vascular causada por la activacion de neutrofilos. Las vitaminas pueden acortar efectivamente la duracion del resfriado comun. En condiciones extremas (atletas, esquiadores, trabajadores de arte, ejercicios militares), puede prevenir eficazmente el resfriado comun. Y si la vitamina $\mathrm{C}$ tambien tiene un cierto efecto protector en los pacientes con influenza, solo unos pocos estudios han demostrado que la deficiencia de vitamina $\mathrm{C}$ esta relacionada con un mayor riesgo y gravedad de las infecciones por influenza a pesar de ello aunque no hay evidencia fuerte de su eficacia y seguridad en infeccion pulmonar por COVID-19. (19)

Actualmente en El Salvador se recomienda el uso de vitamina C en dosis 1.5 g IV cada 6 horas como parte del esquema de tratamiento para pacientes con neumonia grave y neumonia grave con ventilacion mecanica asociada a Shock refractario a catecolaminas y con uso de esteroide. (7)

\section{CONCLUSIONES:}

- Ninguna droga ha sido aprobada por la FDA para su uso en la prevencion, tratamiento, mitigacion o curacion del COVID-19

- La utilidad de los farmacos actualmente empleados en el tratamiento de la enfermedad por COVID-19 se basa en datos descritos en series observacionales y en datos anecdoticos en base a evidencia in vitro o extrapolada.

Actualmente se estan evaluando varios tratamientos para COVID-19. Aunque algunos de estos tratamientos estan clinicamente disponibles para otras indicaciones, su uso para COVID-19 sigue siendo motivo de investigacion

\section{BIBLIOGRAFIA:}

1. Wang M, Cao R, Zhang L, Yang X, Liu J, Xu M, et al. Remdesivir and chloroquine effectively inhibit the recently emerged novel coronavirus (2019-nCoV) in vitro. Cell Res. 2020;30(3):269-71.

2. Agostini ML, Andres EL, Sims AC, Graham RL, Sheahan TP, Lu X, et al. Coronavirus Susceptibility to the Antiviral Remdesivir (GS-5734) Is Mediated by the Viral Polymerase and the Proofreading Exoribonuclease. mBio. 06 de 2018;9(2).

3. Beigel JH, Tomashek KM, Dodd LE, Mehta AK, Zingman BS, Kalil AC, et al. Remdesivir for the Treatment of Covid-19 - Preliminary Report. N Engl J Med. 22 de mayo de 2020;

4. Ritonavir Dosificacion, Efectos secundarios [Internet]. infoSIDA. [citado 11 de junio de 2020]. Disponible en: https://infosida.nih.gov/drugs/244/ritonavir/0/patient

5. Chu CM, Cheng VCC, Hung IFN, Wong MML, Chan KH, Chan KS, et al. Role of lopinavir/ritonavir in the treatment of SARS: initial virological and clinical findings. Thorax. 1 de marzo de 2004;59(3):252-6.

6. Dong L, Hu S, Gao J. Discovering drugs to treat coronavirus disease 2019 (COVID-19). Drug Discov Ther. 2020;14(1):58-60.

7. Lineamientos tecnicos para la atencion clinica de personas con COVID-19. Ministerio de Salud (MINSAL) [Internet]. Disponible en: http://asp.salud.gob.sv/regulacion/pdf/lineamientos/lineamientostecnicosparalaatencionclinicadepersonasconCOVID19TE. 
8. Lopinavir/Ritonavir and Other HIV Protease Inhibitors | Coronavirus Disease COVID-19 [Internet]. COVID-19 Treatment Guidelines. [citado 12 de junio de 2020]. Disponible en: https://www.covid19treatmentguidelines.nih.gov/antiviral-therapy/lopinavir-ritonavir-and-other-hivprotease-inhibitors/

9. Hoja informativa para proveedores de atencion medica autorizacion de uso urgente (EUA) de fosfato de cloroquina proporcionado por la reserva estrategica nacional para el tratamiento del covid19 en ciertos pacientes hospitalizados [Internet]. [Citado 12 de junio de 2020]. Disponible en: https://www.fda.gov/media/137437/download

10. Wong CK, Lam CWK, Wu AKL, Ip WK, Lee NLS, Chan IHS, et al. Plasma inflammatory cytokines and chemokines in severe acute respiratory syndrome. Clin Exp Immunol. abril de 2004;136(1):95-103.

11. Qin Y-Y, Zhou Y-H, Lu Y-Q, Sun F, Yang S, Harypursat V, et al. Effectiveness of glucocorticoid therapy in patients with severe coronavirus disease 2019: protocol of a randomized controlled trial. Chinese Medical Journal. 5 de mayo de 2020;133(9):1080-1086.

12. Bosseboeuf E, Aubry M, Nhan T, de Pina JJ, Rolain JM, Raoult D, et al. Azithromycin Inhibits the Replication of Zika Virus. J Antivir Antiretrovir [Internet]. 2018 [citado 14 de junio de 2020];10(1). Disponible en: https://www.omicsonline.org/open-access/azithromycin-inhibits-thereplication-of-zika-virus-1948-5964-1000173-99806.html

13. Zhou F, Yu T, Du R, Fan G, Liu Y, Liu Z, et al. Clinical course and risk factors for mortality of adult inpatients with COVID-19 in Wuhan, China: a retrospective cohort study. The Lancet. marzo de 2020;395(10229):1054-62. 14. Rosenberg ES, Dufort EM, Udo T, Wilberschied LA, Kumar J, Tesoriero J, et al. Association of Treatment With Hydroxychloroquine or Azithromycin With In-Hospital Mortality in Patients With COVID-19 in New York State. JAMA. 11 de mayo de 2020; 15. Bloch EM, Shoham S, Casadevall A, Sachais BS, Shaz B, Winters JL, et al. Deployment of convalescent plasma for the prevention and treatment of COVID-19. J Clin Invest. 01 de 2020;130(6):2757-65.

16.lineamientostecnicosparausodeplasmaconvalecienteenpacientesconCOVID19-

Acuerdo821_v2.pdf [Internet]. [citado 14 de junio de 2020]. Disponible en:http://asp.salud.gob.sv/regulacion/pdf/lineamientos/lineamientostecnicosparausodeplasmaconvalecienteenpacientesconC Acuerdo821_v2.pdf

17. Dumont LJ, Cancelas JA, Maes LA, Rugg N, Whitley P, Herschel L, et al. The bioequivalence of frozen plasma prepared from whole blood held overnight at room temperature compared to fresh-frozen plasma prepared within eight hours of collection. Transfusion. marzo de 2015;55(3):476-84.

18. Grant WB, Lahore H, McDonnell SL, Baggerly CA, French CB, Aliano JL, et al. Evidence that Vitamin D Supplementation Could Reduce Risk of Influenza and COVID-19 Infections and Deaths. Nutrients. 2 de abril de 2020;12(4). 